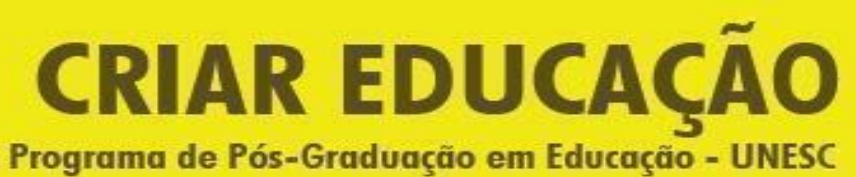

Revista do Programa de Pós-Graduação em Educação - UNESC

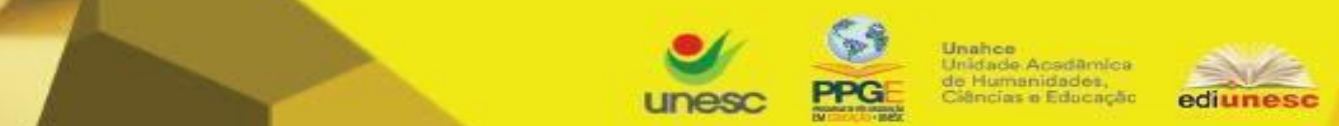

Criar Educação, Criciúma, v. 9, ㄲo2, Edição Especial 2020.- PPGE - UNESC - ISSN 2317-2452

\title{
DIMENSÕES DO MODELO FELDER-SILVERMAN PREDOMINANTES NO ESTILO DE APRENDIZAGEM DE ESTUDANTES DE ADMINISTRAÇÃO
}

\author{
Janine Bertelli ${ }^{1}$ \\ Juliana Matte ${ }^{2}$ \\ Rosani Elisabete Graebin ${ }^{3}$ \\ Pelayo Munhoz Olea ${ }^{4}$
}

\begin{abstract}
RESUMO
O estilo de aprendizagem tem papel significativo na adaptação da metodologia utilizada pelo professor na sala de aula, uma vez que cada aluno tem suas preferências na maneira de receber e processar as informações na construção do conhecimento. O estudo objetivou identificar o estilo de aprendizagem predominante entre os alunos do curso de Administração de uma Instituição de Ensino Superior particular na região dos Campos de Cima da Serra, no Rio Grande do Sul. O estudo é quantitativo, com aplicação de questionário como ferramenta. Participaram da pesquisa 116 alunos. O resultado apresentou estudantes, na sua maioria, ativos, sensoriais, visuais e sequenciais, proporcionando a utilização de métodos mais adequados para a aprendizagem, como apresentações, vídeos, oficinas e seminários.
\end{abstract}

Palavras-chave: Estilos de aprendizagem. Felder-Soloman. Administração.

\section{DIMENSIONS OF THE FELDER-SILVERMAN MODEL PREDOMINANT IN THE ADMINISTRATION STUDENT LEARNING STYLE}

\author{
ABSTRACT \\ The learning style plays a significant role in the adaptation of the methodology used by the \\ teacher in the classroom, since each student has their preferences in the way of receiving and \\ processing information in the construction of knowledge. The study aimed to identify the \\ predominant learning style among the students of the Administration course of a private Higher \\ Education Institution in the region of Campos de Cima da Serra, Rio Grande do Sul, Brazil. \\ The study is quantitative, with a questionnaire as a tool. 116 students participated in the study. \\ The result showed students, mostly active, sensorial, visual and sequential, providing the use

\footnotetext{
1 Mestra em Administração pela Universidade de Caxias do Sul - UCS; janine bert@hotmail.com

2 Doutoranda em Administração no Programa de Pós-Graduação em Administração - PPGA da Universidade de Caxias do Sul - UCS com bolsa FAPERGS/CAPES. Mestre em Administração no Programa de Pós-Graduação em Administração - PPGA da Universidade de Caxias do Sul - UCS; ju.cxs1@gmail.com

${ }^{3}$ Mestra em Administração pela Universidade de Caxias do Sul - UCS; rosanigraebin@hotmail.com

4 Pós-Doutorado em Gestão Ambiental pela Universidad de Extremadura, UEX, Espanha, Bolsista do

Programa de Intercâmbio de Formação de Investigadores entre a União Européia e a América Latina, Programa ALFA2 (América Latina Formación Académica) da Rede Jean Mermoz/ESPAÑA.

Doutorado em Administração e Direção de Empresas pela Universitat Politècnica de Catalunya,
} \\ ETSEIB/UPC.Universidade Federal de Rio Grande (FURG); pelayo.olea@gmail.com
}




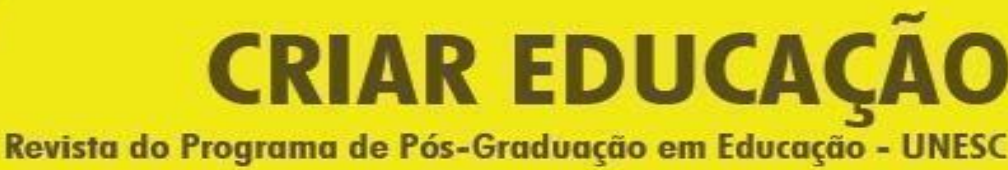

Criar Educação, Criciúma, v. 9, №2, Edição Especial 2020.- PPGE - UNESC - ISSN 2317-2452

of more appropriate methods for learning, such as presentations, videos, workshops and seminars.

Key-words: Learning style. Felder-Soloman. Administration.

\section{INTRODUÇÃO}

Observa-se nas pessoas preferências quanto à forma de apropriar-se das informações, processá-las e construir novos conhecimentos. Este processo pode ser entendido como estilos de aprendizagem (CURY, 2000). Para Silva (2006, p. 45), "os estilos de aprendizagem estão relacionados à forma particular de adquirir conhecimentos, habilidades ou atitudes através da experiência ou anos de estudo".

Os estilos de aprendizagem, ainda de acordo com Lopes (2002, p. 2), "podem ser utilizados para prever tipos de estratégias ou métodos de instruções mais efetivos a determinado aluno ou grupo de estudantes". Quando se apresenta sempre o mesmo procedimento para ensinar, com os mesmos tópicos, os mesmos exercícios, independente do que é interessante ou não para o discente, sem verificar seu estilo de aprendizagem, acaba por não contemplar todas as preferências no modo de aprender dos alunos (DIAS; SAUAIA; YOSHIZAKI, 2013). Este é um dos aspectos que leva à falta de sintonia entre o modo de ensinar e o estilo de aprender dos estudantes, o que acaba prejudicando o desempenho destes no seu percurso acadêmico e profissional, uma vez que os discentes são colocados em uma situação de passividade, ao mesmo tempo em que os professores se deparam com o baixo desempenho e a falta de interesse dos mesmos (SILVA, 2006). Conhecer o estilo dos alunos aprenderem proporciona a utilização de métodos mais adequados para a aprendizagem, oportunizando a adaptação dos métodos utilizados.

Portanto, o objetivo da pesquisa é identificar o estilo de aprendizagem predominante entre os alunos do curso de Administração de uma Instituição de Ensino Superior particular na região dos Campos de Cima da Serra, no Rio Grande do Sul. A pesquisa é quantitativa, com aplicação de questionário como ferramenta para a obtenção dos dados. A pesquisa teve 116 respondentes válidos. Para a identificação 


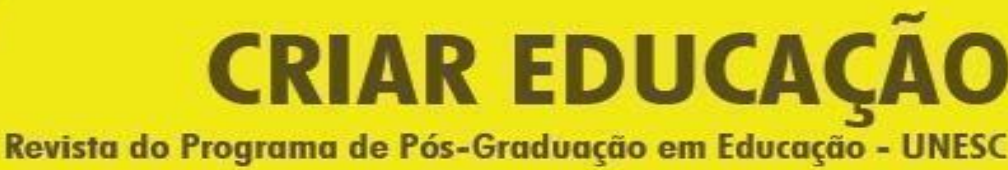

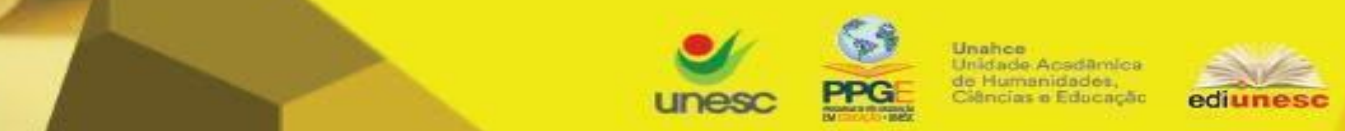

Criar Educação, Criciúma, v. 9, n²2, Edição Especial 2020.- PPGE - UNESC - ISSN 2317-2452

dos estilos de aprendizagem, foi utilizado o Índice de Estilos de Aprendizagem de Felder-Soloman (ILS).

A justificativa para este estudo se dá em função era da informação e da personalização de produtos e serviços e a atividade educacional não pode esperar para sobreviver, posicionando-se como uma ilha, abraçada à Era Industrial, da produção em massa (SCHWAHN; MCGARVEY, 2012). Como resultado, atualmente, a regra, nos cursos e disciplinas do ensino superior, é oferecer sempre o mesmo processo de aprendizagem, com o mesmo conteúdo, os mesmos exercícios para todos os estudantes, independentemente de seus talentos, interesses e desinteresses pessoais, pontos fortes, fracos ou seus estilos de aprendizagem (DIAS; SAUAIA; YOSHIZAKI, 2013). Dessa forma, o estudo visa, a partir da identificação dos estilos de aprendizagem, alertar os docentes para a renovação das formas de aprendizagem, com o intuito de facilitar e otimizar o conteúdo recebido pelo aluno.

$\mathrm{O}$ artigo se inicia com o referencial teórico de estilos de aprendizagem e os modelos de aprendizagem, permeando com a metodologia, análise dos dados e finalizando com as considerações finais.

\section{REFERENCIAL TEÓRICO}

\section{Estilos de aprendizagem}

Encontra-se na literatura uma variedade de definições para o que são estilos de aprendizagem. Abaixo, elenca-se algumas definições de estilos de aprendizagem e seus respectivos autores. Estas definições foram extraídas dos trabalhos de Lopes (2002), Silva (2006) e Oliveira (2012), conforme mostra o Quadro 1 a seguir, ordenado em ordem cronológica.

Quadro 1 - Conceitos de estilos de aprendizagem 
Revista do Programa de Pós-Graduação em Educação - UNESC

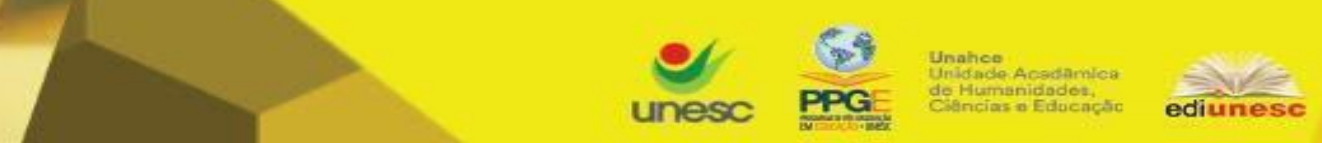

Criar Educação, Criciúma, v. 9, ํㅡ, Edição Especial 2020.- PPGE - UNESC - ISSN 2317-2452

Gregorc (1979): "Estilo de aprendizagem consiste em comportamentos distintos que servem como indicadores da maneira como uma pessoa aprende e se adapta ao seu ambiente" (LOPES, 2002, p.37).

Hunt (1979): "A definição de estilo de aprendizagem baseia-se nas condições educativas com as quais o aluno está em melhor situação para aprender, ou que estrutura necessita 0 aluno para aprender melhor" (SILVA, 2006, p. 46).

Ford (1981): "Estilo de aprendizagem como uma reação estratégica adaptativa para uma situação de aprendizagem particular, cuja intensidade depende, igualmente, de fatores como o nível de interesse, de ansiedade ou mais com estilos estáveis vinculados às características de personalidade e motivação" (LOPES, 2002, p.37).

Butler (1982): "Concebe estilos de aprendizagem como o significado natural da forma como uma pessoa, efetiva e eficientemente, compreende a si mesma, o mundo e a relação entre ambos. Indica uma maneira distinta do aluno se aproximar de um projeto ou episódio de aprendizagem, independentemente da inclusão de uma decisão explícita ou implícita por parte deste" (SILVA, 2006, p. 46).

Keefe (1982): "Os estilos de aprendizagem são constituídos por traços cognitivos, afetivos e fisiológicos, que funcionam como indicadores relativamente estáveis da forma como os alunos percebem, interagem e respondem ao ambiente de aprendizagem" (OLIVEIRA, 2012, p.27).

Schmeck (1982): "Estilo de aprendizagem é o estilo que um indivíduo manifesta quando se confronta com uma tarefa de aprendizagem específica. E, também, uma predisposição do aluno em adotar uma estratégia particular de aprendizagem, independentemente das exigências das tarefas" (LOPES, 2002, p.37).

Butler (1982): "Concebe estilos de aprendizagem como o significado natural da forma como uma pessoa, efetiva e eficientemente, compreende a si mesma, o mundo e a relação entre ambos. Indica uma maneira distinta do aluno se aproximar de um projeto ou episódio de aprendizagem, independentemente da inclusão de uma decisão explícita ou implícita por parte deste" (SILVA, 2006, p. 46).

Keefe (1982): "Os estilos de aprendizagem são constituídos por traços cognitivos, afetivos e fisiológicos, que funcionam como indicadores relativamente estáveis da forma como os alunos percebem, interagem e respondem ao ambiente de aprendizagem" (OLIVEIRA, 2012, p.27).

Schmeck (1982): "Estilo de aprendizagem é o estilo que um indivíduo manifesta quando se confronta com uma tarefa de aprendizagem específica. E, também, uma predisposição do aluno em adotar uma estratégia particular de aprendizagem, independentemente das exigências das tarefas" (LOPES, 2002, p.37).

Fuhrman e Grasha (1983): "Estilos de aprendizagem como interação social, descrevendo os diferentes papéis utilizados pelos alunos na sala de aula na interação com seus pares, professores e conteúdo do curso" (LOPES, 2002, p.37).

Kocinski (1984): "A noção de estilos de aprendizagem como um importante componente da teoria de Jung dos tipos psicológicos" (LOPES, 2002, p.37).

Dunn (1986): "Estilos de aprendizagem são as condições através das quais os indivíduos começam a concentrar-se, absorver, processar e reter informações e habilidades novas e difíceis" (SILVA, 2006, p. 46).

Fisher e Fisher (1987): "Estilo de aprendizagem como a qualidade presente na estratégia de aprendizagem que retorna ao comportamento durante o processo" (OLIVEIRA, 2012, p.27).

Entwistle (1988): "Estilo de aprendizagem é como uma orientação do indivíduo para a aprendizagem, ou seja, a consistência na abordagem que um indivíduo demonstra na realização de tarefas específicas de aprendizagem" (LOPES, 2002, p.37).

Smith (1988): "Os estilos de aprendizagem são como os modelos característicos pelos quais um indivíduo processa a informação, sente e se comporta nas situações de aprendizagem" (SILVA, 2006, p. 46).

DeBello (1990): "Estilo de aprendizagem é a maneira pela qual a pessoa absorve, processa e retêm a informação" (SILVA, 2006, p. 48).

Alonso, Gallego e Honey (1994): "Concebem estilos de aprendizagem como conclusões as quais os seres humanos chegam acerca da forma como atuam as pessoas, abarcando dois níveis: 0 
sistema total do processamento do pensamento e as qualidades peculiares da mente utilizadas para estabelecer laços com a realidade" (OLIVEIRA, 2012, p.26).

Taylor (1998): "O estilo de aprendizagem de cada um está tão profundamente enraizado no indivíduo que ele acredita que o seu estilo é o mais eficiente quando está lidando com outras pessoas" (OLIVEIRA, 2012, p.27).

Sarasin (1999): "Estilo de aprendizagem pode ser definido como certo padrão específico de comportamento e/ou desempenho segundo o qual o indivíduo toma novas informações e desenvolve novas habilidades e o processo pelo qual o indivíduo mantém novas informações ou novas habilidades" (OLIVEIRA, 2012, p.26).

Barreto (2000): "O estilo de aprender é um conceito também muito importante para os professores porque repercute em sua maneira de ensinar" (OLIVEIRA, 2012, p.27).

Campbell, Campbell e Dickinson (2000): "Estilos de aprendizagem referem-se às diferenças individuais na maneira como a informação é compreendida, processada e comunicada" (SILVA, 2006, p.48).

Fonte: adaptado de Lopes (2002), Silva (2006) e Oliveira (2012).

Mesmo com uma quantidade diversificada de definições de estilos de aprendizagem, estas definições não se excluem, e sim, se complementam, cada uma de acordo com a sua abordagem teórica de referência, para buscar expressar como se dá o processo de aprendizagem (LOPES, 2002; SILVA, 2006). Pode-se observar que em todas essas definições os autores relacionam estilos de aprendizagem a modos (comportamentos) característicos de aprendizado, ou seja, à forma como as pessoas interagem com as condições, ambientes ou estruturas sob as quais se processa a aprendizagem (SILVA, 2006, p. 48).

Para Lopes (2002), conhecer as preferências dos alunos para aprender serve como parâmetro para o planejamento estratégico das ações pedagógicas do professor. Assim, professores que transmitem o conteúdo através do seu estilo predominante de ensinar, consequentemente, acabam privilegiando uma das dimensões em detrimento da outra, o que acarreta o favorecimento dos alunos que possuem as mesmas dimensões do professor para a aprendizagem (CURY, 2000).

É preciso também atentar para o cenário em que o professor leva em consideração somente o estilo de aprender preferido dos estudantes, gerando uma situação em que estes não irão desenvolver a amplitude mental necessária para alcançar o máximo potencial em seu desenvolvimento, uma vez que ficarão atrelados somente ao seu estilo preferencial de aprendizagem, o que pode vir a dificultar o seu desempenho profissional futuro, frente às inúmeras situações cotidianas ou exigências do trabalho (LOPES, 2002). O bom ensino é aquele que proporciona o 
ensino para todo o grupo de alunos, isto é, que abrange todas as dimensões da aprendizagem, para que estes capturam as informações (CURY, 2000). Portanto, atender a todas estas demandas dos diferentes estilos de aprendizagem torna-se um desafio para os professores (SILVA et al., 2012).

\section{Modelos de Avaliação dos Estilo de Aprendizagem}

$\mathrm{Na}$ década de 1960, Ausubel $(1980,2003)$ propôs a sua Teoria da Aprendizagem Significativa. Essa teoria propõe que os conhecimentos prévios dos alunos sejam valorizados, para que possam construir estruturas mentais utilizando, como meio, mapas conceituais que permitem descobrir e redescobrir outros conhecimentos, caracterizando, assim, uma aprendizagem prazerosa e eficaz (PELIZZARI, 2002). Ausubel $(1980,2003)$ enfatiza a aprendizagem de significados como aquela mais relevante para seres humanos. Uma de suas contribuições é marcar claramente a distinção entre aprendizagem significativa e a aprendizagem mecânica, o qual a significativa ressalta que o aprendiz transforma o significado lógico do material pedagógico em significado psicológico. Já a mecânica, ou memorística, se dá com a absorção literal e não substantiva do novo material. Como o esforço necessário para a aprendizagem mecânica é muito menor, ela torna-se utilizada em demasia quando os alunos se preparam para exames escolares (AUSUBEL, 1980).

Em razão do surgimento de diversas abordagens para os estilos de aprendizagem e suas diversas classificações desenvolvidas a partir de interesses nas diferenças individuais de aprendizagem, pesquisadores das áreas da educação e psicologia começaram a classificar os entendimentos em categorias ou inventários, com a finalidade de organizar o desenvolvimento desta linha de pesquisa. Assim, surgiram os inventários de estilos de aprendizagem ou learning styles inventories (LSI), instrumentos para a avaliação desses estilos (SILVA, 2006). Assim, os instrumentos utilizados para a avaliação dos estilos de aprendizagem permitem o levantamento do perfil dos estilos dos alunos com a indicação dos prováveis pontos fortes e possíveis tendências ou hábitos que poderiam estar conduzindo a dificuldades na vida acadêmica (LOPES, 2002, p.4). 
Para este trabalho, serão apresentados os modelos de Kolb e Myers-Briggs, uma vez que estes influenciaram o Modelo de Felder-Silverman. Dentre as cinco dimensões, duas são reproduções de aspectos dos modelos de Kolb e Myers-Briggs. A dimensão percepção (sensorial/intuitivo) faz analogia à percepção de Kolb e de Myers-Briggs; já a dimensão (ativo/reflexivo) apresenta-se no modelo de Kolb. A estas duas dimensões, Felder e Silverman acrescentaram as dimensões entrada ou retenção, organização e compreensão (SILVA, 2006).

Modelo de aprendizagem de Kolb

David Kolb desenvolveu a Teoria da Aprendizagem Experiencial (ELT), com base nas abordagens teóricas de Jonhn Dewey, Kurt Lewin e Jean Piaget, em que o conhecimento é adquirido através da transformação da experiência, isto é, é o processo de aprendizagem que ocorre com base na própria experiência do aluno (SILVA et al., 2012). Kolb descreveu que a teoria da abordagem experiencial detinhase numa perspectiva integrada e holística que agrupava a experiência, a percepção, a cognição e o comportamento" (SILVA et al., 2012). É o conhecimento proporcionando o desenvolvimento do estudante (CERQUEIRA, 2000).

Kolb (1984) menciona o processo de aprendizagem é cíclico e passa por quatro etapas de desenvolvimento o qual é chamado de ciclo de aprendizagem experiencial, que está baseado em um ciclo de aprendizagem composto pelas seguintes fases:

a) experiência concreta: aprender experimentando;

b) observação reflexiva: aprender observando, avaliando;

c) conceituação abstrata: aprender pensando, projetando; e

d) experimentação ativa: aprender fazendo, executando (SILVA et al., 2012).

A aprendizagem irá demandar do aluno a combinação destas fases, as quais poderão evoluir para os estilos, os quais caracterizam a individualidade do aluno (LOPES, 2002). Após este estudo, o modelo de Kolb apontou duas dimensões distintas de aprendizagem: a percepção e o processamento. Na dimensão da percepção, alguns estudantes percebem melhor a informação por meio de 


\section{CRIAR EDUCACC̃̃o}

\section{(1)

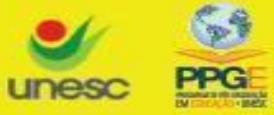 \\ Unahace

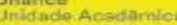 \\ PPG

Criar Educação, Criciúma, v. 9, no2, Edição Especial 2020.- PPGE - UNESC - ISSN 2317-2452

experiências concretas, ao passo que outros percebem melhor através de conceitos mentais ou visuais (abstração), caracterizando a oposição: experiência concreta versus conceituação abstrata (EC - CA). Já para a dimensão do processamento, alguns estudantes processam melhor a informação fazendo experimentação com ela e outros a processaram melhor através da observação reflexiva, constituindo a oposição: experimentação ativa versus observação reflexiva (EA - OR) (LOPES, 2002; SILVA, 2006). Dessas duas dimensões derivam as 4 categorizações dos estilos de aprendizagem e que apresentam as seguintes características, conforme descrito no Quadro 2.

Quadro 2 - Categorias e características dos estilos de aprendizagem

\begin{tabular}{|c|l|}
\hline Categorias & \multicolumn{1}{|c|}{ Características } \\
\hline Tipo Divergente (EC - OR) & $\begin{array}{l}\text { Preferem aprender pela experiência concreta e observação } \\
\text { reflexiva. Analisam as situações sob diferentes pontos de vista } \\
\text { e as relacionam em um todo organizado. Com este tipo de } \\
\text { aluno, o professor deve ter perfil motivador. }\end{array}$ \\
\hline Tipo Assimilador (OR - CA) & $\begin{array}{l}\text { Preferem aprender por observação reflexiva e conceituação } \\
\text { abstrata. São hábeis em criar modelos teóricos, mas não com } \\
\text { seu uso prático. Com este tipo de aluno, o professor deve ter } \\
\text { perfil especialista. }\end{array}$ \\
\hline Tipo Convergente (CA - EA) & $\begin{array}{l}\text { Preferem aprender pela conceituação abstrata e experiência } \\
\text { ativa. Aprendem por ensaio erro, através da aplicação prática } \\
\text { das ideias. Com este tipo de aluno, o professor deve ter perfil } \\
\text { treinador. }\end{array}$ \\
\hline Tipo Acomodador (EA - EC) & $\begin{array}{l}\text { Preferem aprender pela experiência ativa e experiência } \\
\text { concreta. Aprendem fazendo coisas e enfrentando desafios. } \\
\text { Com este tipo de aluno, o professor deve maximizar } \\
\text { oportunidades para os alunos descobrirem coisas por eles } \\
\text { mesmos. }\end{array}$ \\
\hline
\end{tabular}

Fonte: adaptado de Lopes (2002) e Silva (2006).

Para Kolb (1984, p. 24), o estilo de aprendizagem é como "um estado duradouro e estável que deriva de configuração consistente das interações entre o indivíduo e o seu meio ambiente". O objetivo de Kolb era, a partir das habilidades dos alunos para sentir, pensar, fazer e observar, identificar seu estilo de aprendizagem (SILVA et al., 2014).

Modelo indicador de tipos Myers-Briggs - MBTI 
O desenvolvimento do método MBTI (Indicador de Tipos de Myers-Briggs) por Katharine Briggs e sua filha Isabel Myers, é baseado nos tipos psicológicos de Carl Jung, para verificar os diferentes tipos de personalidades (SILVA, 2006; RODRIGUES, 2008). De acordo com a teoria de Jung, existem dois tipos marcantes de pessoas: as extrovertidas, voltadas mais para o mundo externo, e as introvertidas cujo interesse flui para o mundo interno (LOPES, 2002). Sobre as ideias de Jung, Briggs e Myers também acrescentaram, para a identificação dos tipos psicológicos, a escolha entre uma atitude perceptiva e uma atitude julgadora com relação ao modo de viver e se relacionar com o mundo exterior. Para Briggs e Myers, a personalidade estrutura-se pelas quatro preferências que dizem respeito à atitude e ao uso da percepção e do julgamento (SILVA, 2006).

De acordo com Lopes (2002), o inventário MBTI difere os estudantes de acordo com as suas preferências na escala composta de quatro dimensões:

a) orientação para vida (extrovertido/introvertido);

b) percepção (sensorial/intuitivo);

c) abordagens contratantes ao julgamento (reflexivos/sentimentais); e

d) orientação em relação ao mundo externo (julgadores/perceptivos).

O inventário Myers-Briggs Type Indicator (MBTI) classifica os estudantes de acordo com suas preferências na escala composta das quatro dimensões, através de um questionário composto de 100 questões, as quais avaliam como as pessoas se sentem ou se comportam em determinadas situações. De acordo com o modelo de Myers e Briggs, os estilos de aprendizagem são oito: extrovertidos, introvertidos, sensoriais, intuitivos, reflexivos, sentimentais, julgadores e perceptivos (ROBBINS et al., 2010). O Indicador de Tipos Myers-Briggs apresenta as características para cada tipo apresentadas no Quadro 3.

Quadro 3 - Indicador de tipos Myers-Briggs

\begin{tabular}{l}
\hline Tipo Extrovertido (E): São estudantes expansivos, sociáveis e assertivos. Gostam de experimentar \\
as coisas e buscam interação em grupos. \\
\hline $\begin{array}{l}\text { Tipo Introvertido (I): São estudantes quietos e tímidos, com foco no mundo interno, das ideias. } \\
\text { Pensam sobre as coisas e preferem trabalhar sozinhos. }\end{array}$ \\
\hline $\begin{array}{l}\text { Tipo Sensorial (S): São estudantes pragmáticos e que tem preferência pela ordem. Seu foco é } \\
\text { direcionado para fatos, produtos e detalhes. Mostram-se mais confortáveis com a rotina. }\end{array}$ \\
\hline
\end{tabular}




\begin{abstract}
Tipo Intuitivo (N): São estudantes imaginativos e criativos. Seu foco está voltado para significados e possibilidades, confiam em processos do inconsciente. Prefere trabalhar mais a nível de conceitual e possuem uma visão ampliada das situações. Mostram aversão pela rotina.

Tipo Reflexivo (T): São estudantes objetivos. Tendem a tomar decisões usando a lógica, regras e o raciocínio para lidar com os problemas.

Tipo Sentimental (F): São estudantes subjetivos. Tendem a tomar decisões baseadas em valores, sentimentos e considerações pessoais e humanísticas a respeito do assunto.

Tipo Julgador (J): São estudantes que gostam de ter controle sobre suas ações, planejá-las e controla-las. Preferem seguir agendas.

Tipo Perceptivo (P): São estudantes flexíveis e espontâneos. Procuram se adaptar de acordo com as circunstâncias. Tem tendência a sentirem-se ansiosos e inseguros ao tomarem decisões.

Fonte: adaptado de Robbins et al. (2010).
\end{abstract}

As dimensões e suas possibilidades em cada dimensão são identificadas em cada pessoa e combinadas, resultando em 16 possibilidades de tipos. Os tipos não possuem nomes, são definidos pelas iniciais de cada dimensão que compõe o tipo correspondente. Geralmente, estudantes do tipo Introvertido / Intuitivo / Racional / Julgador (INTJ) são definidos como visionários, donos de mentes originais e com propensão a ter ideias e propósitos próprios, pois são independentes, céticos críticos e determinados. Os estudantes definidos como do tipo Extrovertido / Sensorial / Reflexivo / Julgador (ESTJ) são vistos como organizados, são lógicos, realistas, decididos e gostam de organizar e dirigir atividades. Os estudantes do tipo Extrovertido / Intuitivo / Reflexivo / Perceptivo (ENTP) são idealizadores, inovadores e individualistas e têm preferência por ideias empreendedoras. Assim, com a combinação do tipo dos estudantes dentro de cada dimensão, têm-se as possibilidades de tipos (ROBBINS et al., 2010). Também cabe ressaltar que nenhum tipo é melhor do que o outro, mas diferentes (SENRA, 2009).

\title{
Modelo de Felder-Silverman
}

O modelo original foi elaborado pelo Dr. Richard M. Felder, professor de engenharia Química da Universidade Estadual da Carolina do Norte, juntamente com a Dra. Linda K. Silverman, psicóloga educacional. O professor Felder buscava entender as razões das desistências e repetências dos alunos do curso de Engenharia em que lecionava. O trabalho publicado em 1988, conhecido como Modelo FelderSilverman. Identificou práticas de ensino de acordo com as diferentes necessidades 


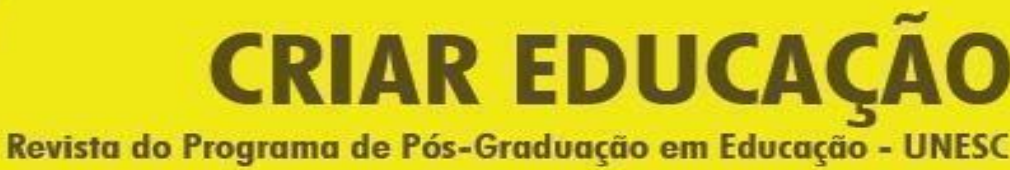

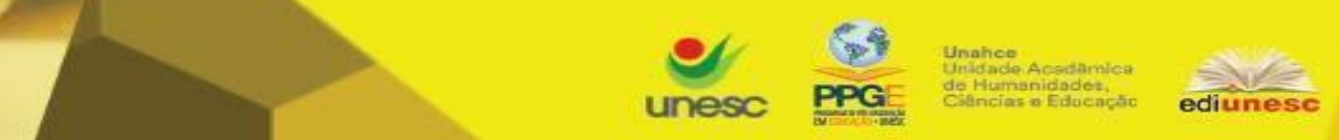

Criar Educação, Criciúma, v. 9, n²2, Edição Especial 2020.- PPGE - UNESC - ISSN 2317-2452

dos alunos - estilos de aprendizagem (SILVA, 2006).

Felder et al. (1988) entendem o processo de aprendizagem como um processo que envolve duas etapas: a recepção e o processamento da informação. $\mathrm{Na}$ etapa da recepção, a informação externa, a qual é captada pelos sentidos, e a informação interna, a qual surge da introspecção, ficam à disposição do indivíduo para que este selecione o que vai ser processado e ignore o restante.

O Modelo de Felder-Silverman (1988) considera cinco dimensões de estilo de aprendizagem:

a) processamento: que pode ser ativo ou reflexivo;

b) percepção: que pode ser sensorial ou intuitiva;

c) entrada/Retenção: que pode ser visual ou verbal;

d) compreensão: que pode ser sequencial ou global; e

e) organização: que pode ser indutiva ou dedutiva.

"Após alguns anos de pesquisa, Felder propôs duas alterações no modelo: omitir a dimensão indutivo-dedutivo e trocar a dimensão visual-ouvinte para visualverbal" (SILVA et al., 2014, p. 4). Essa troca é justificada porque alguns estudantes tendem a focar fatos, datas e algoritmos; outros se sentem mais confortáveis com teorias e modelos matemáticos. Alguns respondem fortemente a formas visuais de informação, gostam de gravuras, diagramas e esquemas; outros aprendem mais através de formas verbais, escrevendo e explicando. Alguns preferem aprender ativamente $\mathrm{e}$ interativamente; outros funcionam melhor introspectivamente $\mathrm{e}$ individualmente (FELDER; FELDER; DIETZ, 2002).

Assim, de acordo com Silva et al. (2012), o autor apresenta quatro processos da informação: percepção, retenção, processamento e organização, conforme descrito:

a) percepção da informação: dimensão sensorial - em que os estudantes aprendem fatos, resolvem problemas e são detalhistas; dimensão intuitiva - os estudantes descobrem possibilidades e relações, lidam com novos conceitos e abstrações e são inovadores;

b) retenção da informação: dimensão visual - onde os estudantes lembram-se do que veem; dimensão verbal, os estudantes aproveitam as explicações orais ou escritas; 


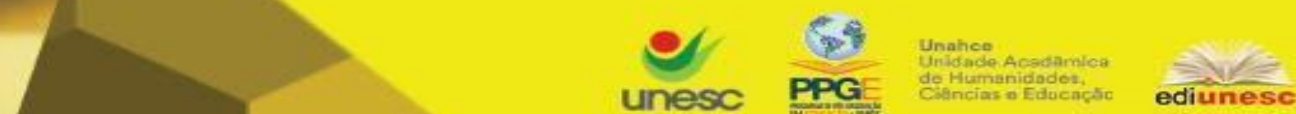

Criar Educação, Criciúma, v. 9, ํㅡ, Edição Especial 2020.- PPGE - UNESC - ISSN 2317-2452

c) processamento da informação: dimensão ativa - onde os estudantes discutem, aplicam conceitos e trabalham em grupos; dimensão reflexiva - os estudantes precisam refletir e preferem trabalhos individuais; e

d) organização da informação: dimensão sequencial - os estudantes aprendem de forma linear e em etapas sequenciais; dimensão global - os estudantes aprendem de forma aleatória formando uma visão do todo e resolvem problemas complexos.

Para avaliar essas dimensões, Felder et al. (1991) desenvolveram, a partir do Modelo de Felder-Silverman, um instrumento chamado ILS (Index of Learning Styles), para identificar os estilos de aprendizagem dos alunos dentro das quatro dimensões propostas. $O$ questionário é composto por 44 questões objetivas, sendo que 11 perguntas são para cada dimensão. O resultado aponta as dimensões dominantes expressas em três escalas:

a) leve, que indica preferência entre ambas as dimensões;

b) moderada, que indica preferência moderada pela dimensão dominante; e

c) forte, indica preferência forte pela dimensão dominante.

A predominância em um dos polos de cada dimensão do modelo não exclui a outra, pois ela pode mudar de acordo com o tipo de conteúdo que está sendo apresentado, como também pode mudar com o passar do tempo (CURY, 2000). De acordo com o autor, os estilos empregados no teste ILS apresentam as seguintes características descritas do Quadro 4.

Quadro 4 - Categorias e características do Modelo de Felder-Silverman

\begin{tabular}{|c|c|}
\hline Categorias & Características \\
\hline Ativo/Reflexivo & $\begin{array}{l}\text { Os alunos ativos preferem aprender aplicando, discutindo ou explicando } \\
\text { para os outros o que foi visto, e gostam de trabalhar em grupo. Para estes } \\
\text { alunos, ficar no somente ouvindo explanações é fatigante. Já o aluno } \\
\text { reflexivo prefere refletir sobre as coisas, processar calmamente as } \\
\text { informações antes de fazer os exercícios. Este aluno prefere trabalhar de } \\
\text { forma individual a trabalhar em grupo. }\end{array}$ \\
\hline Sensitivo/Intuitivo & $\begin{array}{l}\text { Os alunos sensoriais preferem as informações que vêm através dos } \\
\text { sentidos, isto é, aquelas que são observáveis, concretas, fatos, o que vem } \\
\text { da prática, que é perceptível através dos sentidos. Necessitam fazer a } \\
\text { conexão do que foi visto com o mundo real. Tendem a ser metódicos, } \\
\text { preferindo resolver os problemas por meio de testagens. Já os alunos } \\
\text { intuitivos fixam-se mais em conceitos e no que surge através da reflexão e } \\
\text { da imaginação. Gostam de inovações, preferem descobrir as as } \\
\text { possibilidades e as inter-relações entre o que foi visto. Podem ser } \\
\text { descuidados com os detalhes. }\end{array}$ \\
\hline
\end{tabular}




\begin{tabular}{|c|c|}
\hline Visual/Verbal & $\begin{array}{l}\text { Os alunos visuais preferem as informações que vêm por meio visual, isto } \\
\text { é, representações visuais, tais como: diagramas, quadros, cronogramas, } \\
\text { gráficos, esquemas, filmes, demonstrações. Já os alunos verbais preferem } \\
\text { as informações quem vem através da fala e da escrita (materiais para } \\
\text { leitura) transformando o que está escrito em fala. }\end{array}$ \\
\hline Indutivo/Dedutivo & $\begin{array}{l}\text { Os alunos indutivos preferem ver primeiramente os casos particulares (as } \\
\text { observações, os resultados de experiências, os exemplos gráficos ou } \\
\text { numéricos) para depois chegar ao entendimento dos princípios e teorias, } \\
\text { isto é, partem do entendimento do que é específico para o entendimento } \\
\text { do todo, do geral. Já os alunos dedutivos, ao contrário, preferem ter } \\
\text { primeiramente a visão geral das teorias e conceitos para então deduzir as } \\
\text { suas aplicações para os casos específicos. Partem do entendimento do } \\
\text { todo, do geral para o entendimento específico. }\end{array}$ \\
\hline Sequencial/Global & $\begin{array}{l}\text { Os alunos sequenciais gostam de aprender passo a passo, têm o } \\
\text { entendimento do conteúdo de forma linear, gradual e lógica. Têm facilidade } \\
\text { para explicar o seu raciocínio e resolver problemas. Já os alunos globais } \\
\text { aprendem os conteúdos quase que aleatoriamente, de modo não-linear. } \\
\text { São holísticos, necessitam compreender o todo. A informação apresentada } \\
\text { precisa ser relacionada com os seus conhecimentos prévios; ao } \\
\text { compreender o todo, é capaz de resolver rapidamente problemas } \\
\text { complexos, mas sentem dificuldade em explicar os passos de seu } \\
\text { raciocínio. }\end{array}$ \\
\hline
\end{tabular}

Fonte: Felder et al. (1991).

As dimensões dos estilos de aprendizagem dos alunos não são estáticas, isto é, elas podem modificar-se com o tempo, de acordo com as mudanças que ocorrem com o estudante que a carrega, advindas do ambiente educacional em que o mesmo está inserido (LOPES, 2002).

A partir do exposto, realizou-se uma comparação entre os três estilos de aprendizagem, a fim de evidenciar convergências e divergências dos modelos. $\mathrm{Na}$ categoria Divergente, de Kolb, há características comuns do reflexivo e introvertido de Myers-Briggs e de Felder-Silverman, a partir da reflexão inicial feita pelo aluno para a aprendizagem. A categoria assimilador de Kolb se assimila com as categorias de intuitivo de Myers-Briggs e de Felder-Silverman, cujos discentes que possuem essas características são mais teóricos e abstratos, com maior capacidade para imaginação e criação de modelos teóricos.

Já a categoria convergente de Kolb possui similaridade com o julgador de Myers-Briggs e com o dedutivo e o ativo de Felder-Silverman, pois são estudantes que aprendem partindo da teoria para a aplicação prática. A categoria acomodador de Kolb possui características comuns do extrovertido e sensorial de Myers-Briggs e o 
Revista do Programa de Pós-Graduação em Educação - UNESC

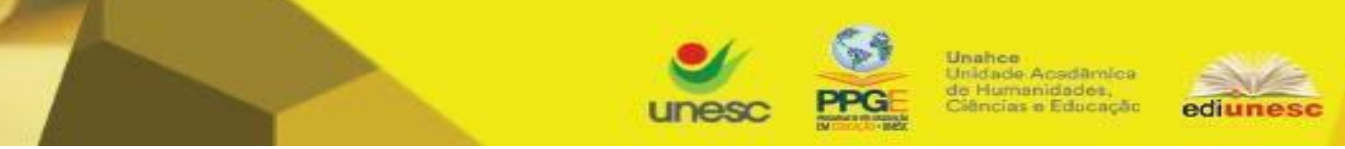

Criar Educação, Criciúma, v. 9, oㅡ, Edição Especial 2020.- PPGE - UNESC - ISSN 2317-2452

sensitivo de Felder-Silverman, uma vez que tipo de aluno aprende diretamente com a prática, com fatos, a partir da observação e experimentação.

Ainda, as categorias sentimental e perceptivo de Myers-Briggs não se assemelha com nenhuma de outro autor, pois a sentimental enfoca a decisão tomada em sentimentos e considerações pessoais e o perceptivo mostra elucida a capacidade de adaptação do discente. E quanto as categorias de sequencial/global e visual/verbal de Felder-Silverman somente mostram formas de aprendizado, mas que podem pertencer a qualquer outra categoria anterior. A sequencial/global é a forma como o aprendizado deve ser exposto, seja por etapas (sequência) ou em sua totalidade, e a visual/verbal identifica a forma como o aluno prefere receber a informação. O Quadro 5 apresenta o comparativo.

Quadro 5 - Comparativo dos estilos de aprendizagem

\begin{tabular}{|l|l|l|}
\hline \multicolumn{3}{|c|}{ Autores e estilos de aprendizagem } \\
\hline Kolb & Myers-Briggs - MBTI & Felder-Silverman \\
\hline Divergente & Reflexivo/Introvertido & Reflexivo \\
\hline Assimilador & Intuitivo & Intuitivo \\
\hline Convergente & Julgador & Dedutivo/Ativo \\
\hline Acomodador & Extrovertido/Sensorial & Sensitivo \\
\hline & Sentimental & \\
\hline & Perceptivo & \\
\hline & & Sequencial/Global \\
\hline & & Visual/Verbal \\
\hline
\end{tabular}

Fonte: elaborado pelos autores (2020).

\section{METODOLOGIA}

A pesquisa possui uma abordagem quantitativa com objetivo exploratório e descritivo. O método de pesquisa quantitativo envolve a coleta de dados, que é submetida para um número significativo de respondentes da população-alvo, a qual se denomina amostra. Após a coleta dá-se a análise dos dados numéricos e aplicação dos testes estatísticos (HAIR Jr. et al., 2005).

Para este estudo, foi utilizado o instrumento ILS desenvolvido por Felder et al. (1991), por conveniência, uma vez que a ferramenta se encontra livremente na Internet. $\mathrm{O}$ referido questionário também pode ser respondido por meio da internet a 


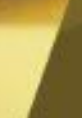

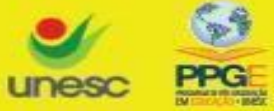 \\ Unahoe.

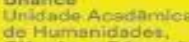 \\ unesc PPG

Criar Educação, Criciúma, v. 9, №2, Edição Especial 2020.- PPGE - UNESC - ISSN 2317-2452

fim de investigar e identificar as preferências de aprendizagem nas dimensões ativo/reflexivo, sensorial/intuitivo, visual/verbal e sequencial/global.

A amostra deste estudo foram os alunos de graduação matriculados no curso de Administração em uma Instituição de Ensino Superior particular localizado nos Campos de Cima da Serra, no Estado do Rio Grande do Sul. A amostra foi determinada pelos critérios de conveniência.

Para identificar o estilo de aprendizagem dos alunos, o instrumento de pesquisa utilizado foi o ILS (Inventário de Estilos de Aprendizagem) de Felder et al. (1991), sendo que as três questões sobre o perfil foram acrescentadas ao instrumento juntamente com as 44 questões estruturadas.

No Modelo Felder-Silverman (1991), os estilos de aprendizagem têm como base escalas de preferência dos estudantes. Segundo Silva et al. (2014), a avaliação da escala pode ser enquadrada em 3 dimensões, de acordo com os escores, conforme demonstrado no Quadro 6.

Quadro 6 - Avaliação dos escores do Modelo Felder-Silverman Escore entre 1 e 3: O estudante possui leve preferência entre ambas dimensões da escala. Escore entre 5 e 7: $O$ estudante tem uma preferência moderada por uma das dimensões e aprenderá mais facilmente se ambiente de ensino favorecer esta dimensão.

Escore entre 9 e 11: O estudante tem uma forte preferência por uma das dimensões da escala, e pode ter dificuldades de aprendizagem em um ambiente que não favoreça esta preferência.

Fonte: adaptado de Silva et al. (2014).

Os dados resultantes da pesquisa surgiram das respostas aos questionários impressos aplicados nas salas de aula em que ocorreram as disciplinas ligadas ao curso de Administração, nos dias 08, 12 e 15 de agosto de 2016, para os alunos que se faziam presentes. Foram respondidos 123 questionários para identificação dos estilos de aprendizagem, correspondendo à totalidade dos alunos presentes nas datas referidas.

No momento da tabulação, percebeu-se que, em sete questionários, haviam perguntas não respondidas. Dessa forma, eles foram excluídos das análises. Portanto, foi considerado para a análise dos dados 116 questionários completamente preenchidos, ou seja, válidos. 
Revista do Programa de Pós-Graduação em Educação - UNESC
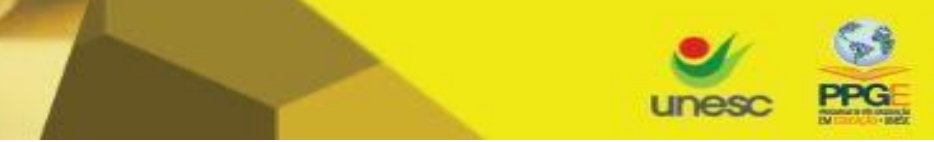

Unahoe

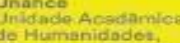

unesc PPG:

Criar Educação, Criciúma, v. 9, oㅡ2, Edição Especial 2020.- PPGE - UNESC - ISSN 2317-2452

Para a análise dos dados foi realizada no software IBM SPSS Statistics 20, através do qual foi realizada a análise de frequências das variáveis do perfil dos estudantes e dos estilos de aprendizagem, sendo que a interpretação foi realizada por meio dos resultados das tabelas descritivas.

\section{ANÁLISE DOS RESULTADOS}

$\mathrm{Na}$ Tabela 1, percebe-se que os estudantes com idade entre 18 a 21 anos representam 43,98 \% dos estudantes, 31,03\% tem entre 22 a 25 anos e 14,65 \% mais de 30 anos. O resultado aponta que $75 \%$ dos estudantes de Administração possuem idade entre 18 e 25 anos.

Tabela 1 - Idade dos respondentes

\begin{tabular}{lcccc}
\hline \multicolumn{1}{c}{ Alternativas } & Frequência & Percentual & $\begin{array}{c}\text { Percentagem } \\
\text { Válida }\end{array}$ & $\begin{array}{c}\text { Percentagem } \\
\text { Cumulativa }\end{array}$ \\
\hline Menos de 17 anos & 02 & 1,72 & 1,72 & 1,72 \\
\hline De 18 a 21 anos & 51 & 43,98 & 43,98 & 45,7 \\
\hline De 22 a 25 anos & 36 & 31,03 & 31,03 & 76,73 \\
\hline De 26 a 29 anos & 17 & 8,62 & 8,62 & 85,35 \\
\hline Mais de 30 anos & 10 & 14,65 & 14,65 & 100,0 \\
\hline Total & 116 & 100,0 & 100,0 & \\
\hline
\end{tabular}

Fonte: elaborada pelos autores (2016).

Observa-se a predominância de estudantes do gênero feminino, que representa $51,73 \%$ e 48,27\% do gênero masculino, conforme ilustrado na Tabela 2 .

Tabela 2 - Gênero dos respondentes

\begin{tabular}{lcccc}
\hline Alternativas & Frequência & Percentual & $\begin{array}{c}\text { Percentagem } \\
\text { Válida }\end{array}$ & $\begin{array}{c}\text { Percentagem } \\
\text { Cumulativa }\end{array}$ \\
\hline Masculino & 56 & 48,27 & 48,27 & 48,27 \\
\hline Feminino & 60 & 51,73 & 51,73 & 100,0 \\
\hline Total & 116 & 100,0 & 100,0 & \\
\hline Fol & & & &
\end{tabular}

Fonte: elaborada pelos autores (2016).

Em relação a região de naturalidade dos estudantes, 67,24 \% são naturais do município de Vacaria, $18,11 \%$ são de outras cidades do estado do Rio Grande do Sul ou do Brasil e 14,65\% são advindos de outras cidades dos Campos de Cima da Serra, conforme ilustrado na Tabela 3. 
Tabela 3 - Origem

\begin{tabular}{lcccc}
\hline \multicolumn{1}{c}{ Alternativas } & Frequência & Percentual & $\begin{array}{c}\text { Percentagem } \\
\text { Válida }\end{array}$ & $\begin{array}{c}\text { Percentagem } \\
\text { Cumulativa }\end{array}$ \\
\hline Vacaria & 78 & 67,24 & 67,24 & 67,24 \\
\hline $\begin{array}{l}\text { Outra cidade dos Campos de } \\
\text { Cima da Serra }\end{array}$ & 17 & 14,65 & 14,65 & 81,89 \\
\hline Outra cidade do RS ou do Brasi & 21 & 18,11 & 18,11 & 100,0 \\
\hline Total & 116 & 100,0 & 100,0 & \\
\hline
\end{tabular}

Fonte: Elaborada pelos autores (2016).

Além de identificar o perfil dos alunos, verificou-se o estilo de aprendizagem em cada dimensão do Modelo Felder-Silverman (1991). Observou-se que as preferências dos alunos em relação ao processamento, a percepção, a retenção e a organização da informação são na dimensão ativo (54\%), sensorial (66\%), visual (64\%) e sequencial (60\%), conforme Tabela 4.

Tabela 4 - Estilo de aprendizagem dos entrevistados

\begin{tabular}{|c|c|c|c|}
\hline Processo & Dimensão & Frequência & Entrevistados (\%) \\
\hline \multirow{2}{*}{ Processamento da Informação } & Ativo & 62 & 54 \\
\hline & Reflexivo & 54 & 46 \\
\hline \multirow{2}{*}{ Percepção da Informação } & Sensorial & 76 & 66 \\
\hline & Intuitivo & 40 & 34 \\
\hline \multirow{2}{*}{ Retenção da Informação } & Visual & 74 & 64 \\
\hline & Verbal & 42 & 36 \\
\hline \multirow{2}{*}{ Organização da Informação } & Sequencial & 70 & 60 \\
\hline & Global & 46 & 40 \\
\hline
\end{tabular}

Fonte: elaborada pelos autores (2016).

Com relação ao processamento da informação, a dimensão reflexiva representou $46 \%$ dos entrevistados. No processo de percepção da informação, a dimensão intuitiva representou $34 \%$ dos entrevistados. No processo de retenção da informação, a dimensão verbal representou $42 \%$ dos entrevistados, e no processo de organização da informação, a dimensão global representou $40 \%$ dos entrevistados. Sendo assim, as dimensões reflexivo, intuitivo, verbal e global também possuem relevante percentual no estilo de aprendizagem.

Ao analisar os 116 questionários, observou-se, a partir do processamento da informação, os estudantes possuem preferências próximas quanto à dimensão ativo e reflexivo, com maior percentual para o ativo (54\%). Quanto ao processo da 
percepção da informação, os alunos sensoriais têm uma preferência acentuada, representando $66 \%$ do total. Na retenção da informação, os alunos visuais têm uma preferência, totalizando $64 \%$ do total dos respondentes. Por fim, quanto à organização da informação, os alunos sequenciais têm uma preferência maior, representando $60 \%$ do total da amostra.

\section{Considerações Finais}

Para Felder et al. (1988), a identificação dos estilos de aprendizagem tem duas aplicações principais: primeiramente, servir para ajudar os professores a adotar uma metodologia para atender a todos os estilos de aprendizagem dos alunos e servir como um norteador para guiá-los sobre a diversidade presente nas salas de aula. Caso o professor ache um grande número de alunos com uma preferência que seja completamente diferente da sua preferência para ensinar, ele poderá rever sua metodologia. A segunda aplicação se relaciona ao aluno que, ao identificar o seu estilo, pode buscar complementação para o que for preciso para melhorar o seu aproveitamento. (FIGUEIREDO; NORONHA; OLIVEIRA NETO, 2008).

Com relação ao objetivo da pesquisa de identificar o estilo de aprendizagem, nos 116 estudantes de Graduação em Administração em uma Instituição de ensino particular no Estado do Rio Grande do Sul, o objetivo foi atingido, uma vez que a pesquisa proporcionou o levantamento das características da população estudada, como também a identificação dos estilos de aprendizagem predominantes, classificando-os quanto ao processo e a dimensão da aprendizagem. Estas informações também podem contribuir para os professores, uma vez que, tendo acesso a este tipo de informação, os mesmos podem rever suas metodologias adotadas em sala de aula.

Os resultados, quanto ao perfil dos alunos respondentes do questionário, identificaram que $75 \%$ dos estudantes de Administração possuem idade entre 18 e 25 anos, com predominância de estudantes do gênero feminino, o qual representa $51,73 \%$ do total pesquisado. Com relação à região de naturalidade dos estudantes, 
$67,24 \%$ são naturais do município de Vacaria e $18,11 \%$ são outras cidades do estado do Rio Grande do Sul ou do Brasil.

Em relação ao estilo de aprendizagem de Felder et al. (1991), os estudantes de Graduação em Administração em sua maioria possuem perfil predominante nas dimensões sensorial e sequencial, com uma preferência moderada para estas dimensões. Sendo assim, os alunos que forem estimulados nestas dimensões aprenderão com mais facilidade se o ambiente de ensino favorecer estas dimensões.

O método utilizado pela pesquisa (ILS) identificou a maioria dos alunos como ativos, sensoriais, visuais e sequenciais. Os resultados encontrados, assemelham-se aos resultados de Felder et al. (1993; 1996; 1998; 2002) quando da aplicação do instrumento para os alunos de Engenharia nos Estados Unidos. Também Lopes (2002), em sua tese, encontrou resultados equivalentes ao aplicar este instrumento para os alunos dos cursos de Ciências Exatas (FIGUEIREDO; NORONHA; OLIVERA NETO, 2008).

A partir dos resultados encontrados nas análises, a metodologia dada em aula deve ser preferencialmente com exposição visual (com utilização de quadros, vídeos) e com aulas práticas, permitindo que o aluno interaja com seus colegas (seminários, apresentações, oficinas). Outro ponto importante é a dimensão sequencial, que faz com que os professores tenham uma rotina na didática, mantendo o foco naquilo que se quer propor, para alcançar e manter a atenção do aluno.

Como limitações da pesquisa, destaca-se que o estudo se limitou a uma Instituição de Ensino Superior particular na região dos Campos de Cima da Serra, no Estado do Rio Grande do Sul. Além disso, para pesquisas futuras, sugere-se a replicação da pesquisa em cursos de Graduação de áreas diversas, sendo relevante para instituições de Ensino Superior o conhecimento e entendimento dos estilos de aprendizagem dos seus alunos, a fim de que possam estruturar as formas de exposição dos conteúdos aos alunos, assim como auxiliar os professores na escolha de sua metodologia de ensino para que consigam atingir o maior número de estudantes possível.

\section{REFERÊNCIAS BIBLIOGRÁFICAS}


AUSUBEL, D. P.; NOVAK, J. D. e HANESIAN, H. Psicologia Educacional. Rio de Janeiro: Interamericana, 1980.

AUSUBEL, D. P. Aquisição e retenção de conhecimentos: Uma perspectiva cognitiva, Lisboa: Plátano, 2003.

CERQUEIRA, T. C. S. Estilos de Aprendizagem em Universitários. 2000. 155 f. Tese (Doutorado em Educação) - Universidade Estadual de Campinas. São Paulo: Campinas, 2000.

CURY, H. N. Estilos de Aprendizagem de Alunos de Engenharia. Anais do XXVII Congresso Brasileiro de Ensino de Engenharia. 2000. Ouro Preto, MG.

DIAS, G. P. P.; SAUAIA, A. C. A.; YOSHIZAKI, H. T. Y. Estilos de aprendizagem Felder-Silverman e o aprendizado com jogos de empresa. Revista de Administração de Empresas, v. 53, n. 5, p.469-484, out. 2013. http://dx.doi.org/10.1590/s0034-75902013000500005.

FELDER, R. M. et al. Index of learning styles. 1991.

FELDER, R. M. et al. Learning and teaching styles in engineering education. Engineering education, v. 78, n. 7, p. 674-681, 1988.

FELDER, R. M.; FELDER, G. N.; DIETZ, E. J. The Effects of Personality Type on Engineering Student Performance and Attitudes. Journal Of Engineering Education, v. 91, n. 1, p. 3-17, jan. 2002. http://dx.doi.org/10.1002/j.21689830.2002.tb00667.x.

FIGUEIREDO, R. S.; NORONHA, C. M. S.; OLIVEIRA NETO, O. J. Estilos de aprendizagem no ensino técnico agropecuário das escolas técnicas federais do Estado de Goiás. Revista Brasileira de Gestão e Desenvolvimento Regional, v. 4, n. 2, 2008.

HAIR Jr., J. F.; ANDERSON, R. E.; TATHAM, R. L.; BLACK, W. C. Análise multivariada de dados. 5. ed. Porto Alegre: Bookman, 2005.

KLINE, R. B. Principles and practice of structural equation modeling. 3 ed. New York: The Guilford Press, 2011.

KOLB, D. A. Experiential learning: experience as the source of learning and development. Englewood Cliffs, New Jersey: Prentice Hall, 1984.

LOPES, W. M. G.ILS - Inventário de Estilos de Aprendizagem de FelderSaloman: investigação de sua validade em estudantes universitários de Belo 
Horizonte. 2002. 96 f. Dissertação (Mestrado em Engenharia da Produção) Universidade Federal de Santa Catarina:Florianópolis, 2002.

OLIVEIRA, D. E. Impacto dos Estilos de Aprendizagem no Desempenho Acadêmico do Ensino de Contabilidade: Uma Análise dos Estudantes da Universidade Federal do Rio Grande do Norte. 2012. 107 f. Dissertação (Mestrado em Ciências Contábeis) - Universidade Federal do Rio Grande do Norte: Natal, 2012.

ROBBINS, S. P.; JUDGE, T. A; SOBRAL, F. Comportamento Organizacional. São Paulo: Pearson Prentice Hall, 2010.

RODRIGUES, M. O. Mapeamento do Estilo de Aprendizagem da Agroindústria de Carne Bovina em Goiás. 2008. 104 f. Dissertação (Mestrado em Agronegócio) Universidade Federal de Goiás. Goiânia: 2008.

SCHWAHN, C; MCGARVEY, B. Inevitable: mass customized learning, learning in the age of empowerment. Independent Publishing, 2012.

SENRA, M. C. S. Os Estilos de Aprendizagem de Felder a partir de Jung. 2009. 111 f. Dissertação (Mestrado em Educação Tecnológica) - Centro Federal de Educação Tecnológica: Belo Horizonte, 2009.

SILVA, Anielson Barbosa da et al. DIMENSÕES DE UM SISTEMA DE APRENDIZAGEM EM AÇÃO PARA O ENSINO DE ADMINISTRAÇÃO. Administração: Ensino e Pesquisa, v. 13, n. 1, p.11-41, 31 mar. 2012. http://dx.doi.org/10.13058/raep.2012.v13n1.97.

SILVA, D. M. O Impacto dos Estilos de Aprendizagem no Ensino de Contabilidade. 2006. 169 f. Dissertação (Mestrado em Controladoria e Contabilidade) - Universidade de São Paulo: Ribeirão Preto, 2006.

SILVA, J. E. A.; GANZER, P. P.; GASPERIN, D.; BIELGEMEYER, U.H.; CAMARGO, M.E.; OLEA, P. M. Estilo de Aprendizagem Acadêmica: Uma Análise com Estudantes de Administração do Ensino superior. XIV Colóquio - CIGU. Florianópolis, 2014. 